\title{
Tertiary Vascular Accesses: Which One do You Control?
}

\section{Rezziki Abdellah*, Abuatayef Taha, Benzirar Adnane and Elmahi Omar}

University of Mohammed the First, Morocco

*Corresponding author: Rezziki Abdellah, Vascular Surgeron, University of Mohammed the First, Oujda, Morocco; E-mail: Dr.rezziki.abdellah@gmail.com Received Date: May 01, 2018; Accepted Date: May 16, 2018; Published Date: May 23, 2018

Copyright: $\odot 2018$ Abdellah $\mathrm{R}$ et al. This is an open-access article distributed under the terms of the Creative Commons Attribution License, which permits unrestricted use, distribution, and reproduction in any medium, provided the original author and source are credited.

\section{Abstract}

Introduction: Vascular access for hemodialysis of choice remains the Arteriovenous Fistulas (AVF) of the Upper Limb, the creation of Lower Limb (LL) arteriovenous fistula is an alternative to the depletion of vascular access sites to the upper limb. The most used surgical technique is the transposition of the femoral vein; recent and poorly known technique.

Material and methods: Through two clinical observations, we report our experience in the creation of exotic vascular approaches (AVF-LL).

Discussion: The complete exhaustion of the veins of both upper limbs and the presence of venous disease occlusive central veins is a major problem in the creation of FAV upper limb, the most commonly used alternative to the lower limb is the transposition of the femoral vein described for the first time in 2000 by Jackson.

Conclusion: The depletion of vascular sites is a delicate problem, hence the interest of knowing how to use the tertiary vascular approaches, particularly fistulas of the lower limb.

Keywords: Arteriovenous fistula; Lower limb; Hemodialysis

\section{Introduction}

Vascular access for hemodialysis of choice remains arteriovenous fistulas (AVF) in the upper limbs [1]. The creation of arteriovenous fistula at the level of the lower limb (AVF-LL) is an alternative to depletion of vascular access sites in the upper limb. The most used surgical technique is the transposition of the femoral vein first described in 2000 by Jackson [2], recent and poorly known technique.

Through two clinical observations, we report our experience in the creation of exotic vascular access.

\section{Material and Methods}

\section{Observation no 1}

40 year old woman, hemodialysis since 2006, who benefited from the 2006 to the 2015 of several vascular access with native AVFs, straight and looped prothetic axillary axillary bypasses, and permanent jugular catheters.

Faced with the exhaustion of the venous capital of the upper limbs, we opted for the creation of a AVF at the level of the right lower limb using the superficialized femoral vein, (Figure 1) this AVF is still functional, in hemodialysis, the fistula presents no problem of flow or hyperpressure which testifies its good permeability (Figures 2 and 3).

\section{Observation no 2}

72 years old woman, hemodialysis since 03 years, admitted for a big right apper limb following the making of a right humeral-cephalic AVF, the patient benefited from a fistulography which objectified a complete occlusion of the central veins not accessible to an endovascular treatment.

A fistula was created in this patient at the level of the right lower limb; using the superficial femoral vein.

There was a postoperative lymphorea that was treated with compression bandages, with good evolution and regression of lymphorea during 2 weeks of treatment.

In hemodialysis, the fistula presents no problem of flow or hyperpressure which testifies its good permeability.

In both cases the primary patency at one year of evolution is evaluated at $100 \%$, without stenosis or thrombosis of the vein.

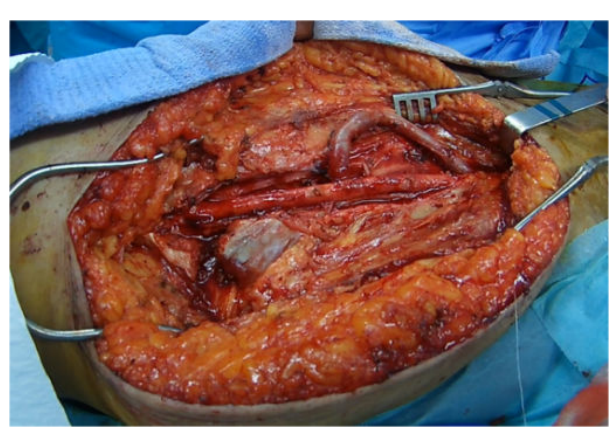

Figure 1: Intraoperative photo of femoral vein superficialized with arteriovenous anastomosis. 


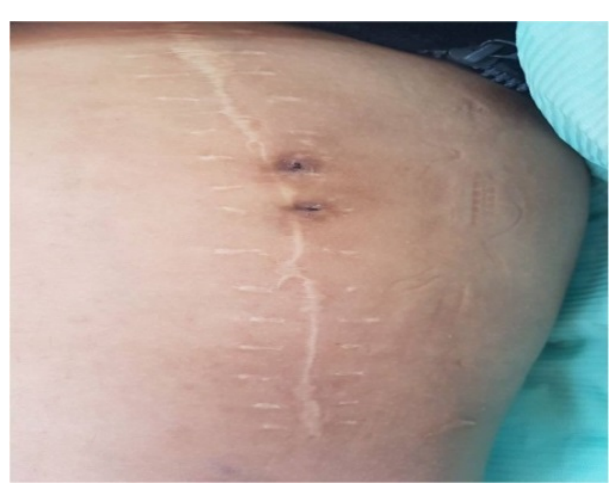

Figure 2: The two puncture sites of the fistula at the level of the right thigh.

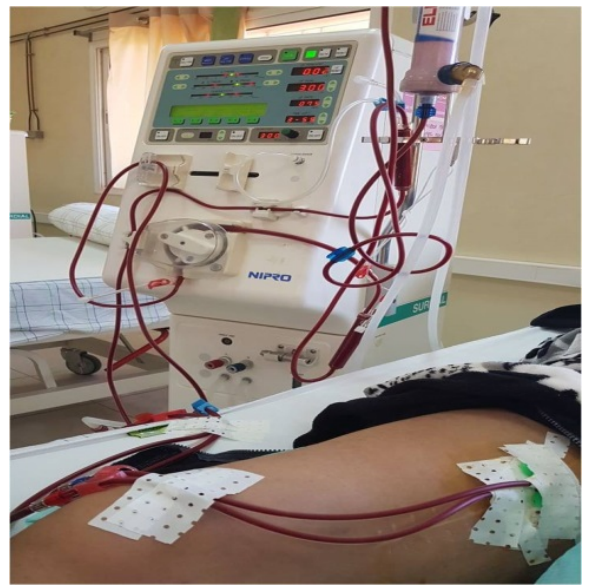

Figure 3: Photo during the hemodialysis session showing the AVF connection of the right MI.

\section{Discussion}

In 1970 George Thomas, conceived the first vascular access of the lower limb with success at the level of the inguinal region [3]. This vascular access at the lower limb level provides a reasonable option when other upper limb access sites are not available. Thomas Shunt was abandoned due to high rate of infection and secondary bleeding [4]. According to the American Society of Nephrology, the preliminaries of an AVF confection of the lower limb are [5]:

- The total exhaustion of the veins of both upper limbs.

- The presence of a vein occlusive disease of the central veins.

- A normal systolic pressure index (ABI) at the lower extremity fistula site.
Surgical technique, transposition of the femoral vein (tVF) for AVF -LL, remains the best compared to other techniques, such as the use of the saphenous vein or arteriovenous graft [6].

Gardman et al. reported low thrombosis in 25 cases with tVF-LL and no case of infection has been reported. A patient was amputated following a compartment syndrome [7].

The main complications reported in the literature have been described in the first two reports concerning tVF, one by Huber et al. [8] and the other by Jackson [2] who are hyper-flow yet heart failure does not develop, and ischemia by hemo-diversion in one patient. Hazinedaroglu et al. [9] reported a series of 15 cases of tVF, five of which had had a wound infection. In our series, there were no major complications; postoperative lymphorea was treated with compression dressings for two weeks with regression of symptoms.

\section{Conclusion}

Continuous monitoring of the vascular access is the best solution to ensure a maximum rate of primary and secondary patency.

Vascular site depletion is a delicate problem hence the interest of knowing how to use the tertiary vascular approaches, particularly fistulas of the lower limb using the superficial femoral vein.

\section{References}

1. Depner T, Daugirdas J (2006) Clinical practice guidelines and clinical practice recommendations for 2006 updates: Hemodialysis adequacy, peritoneal dialysis adequacy and vascular access. Am J Kidney Dis 48: S1e322.

2. Jackson MR (2010) The superficial femoral-popliteal vein transposition fistula: Description of a new vascular access procedure. J Am Coll Surg 191: 581-584.

3. Gibbons CP (2006) Primary vascular access. Eur J Vasc Endovasc Surg 31: 523-529.

4. Freedman BI, Andersson RL, Tuttle AB, Canzanello VJ (1992) The thomas shunt revisited. AM J Kidney dis 11: 45-48.

5. Santoro D, Benedetto F, Mondello P, Pipitò N, Barillà D, et al. (2014) Vascular access for hemodialysis: current perspectives. Int J Nephrol Renovasc Dis 7: 281-284.

6. Parekh VB, Niyyar VD, Vachharajani TJ (2016) Lower extremity permanent dialysis vascular access. Clin J Am Soc Nephrol 11: 1693-1702.

7. Gradman WS, CohenC, Haji-Aghaii M (2001) Arteriovenous fistula construction in the thigh with transposed superficial femoral vein: Our initial experience. J Vasc Surg 30: 968-975.

8. Huber TS, Ozaki CK, Flynn TC, Ross EA, Seeger JM (2000) Use of superficial femoral vein for hemodialysis arteriovenous access. J Vasc Surg 31: 1038-1041.

9. Hazinedaroglu SM, Tu zu ner A, Ayli D, Demirer S, Duman N, et al. (2004) Femoral vein transposition versus femoral loop grafts for hemodialysis: A prospective evaluation. Transplant Proc 36: 65-67. 